\section{II faut vivre longtemps pour devenir un homme}

(Antoine de Saint-Exupéry)
Il n'y a pas bien longtemps, une chirurgienne de 80 ans a été accusée d'avoir osé, à son grand âge, recourir au scalpel et pratiquer une opération. Elle n'a pas été mise en accusation, cependant, pour cela même, mais parce qu'elle avait choisi un mode chirurgical inhabituel. La question s'est alors posée - ce n'est pas étonnant - de savoir combien de temps un médecin peut en fait pratiquer et quand il doit arrêter son activité médicale.

De nombreux libres praticiens, au contraire de leurs confrères salariés, ne cessent pas d'un jour à l'autre toute activité, mais réduisent progressivement leur charge de travail. Il leur arrive souvent de continuer pendant plusieurs années après l'âge de la retraite, sans protestation de nulle part, pour soigner leurs vieux patients malades chroniques. Ces derniers leur en sont très reconnaissants. Je pense que la plupart des généralistes et des spécialistes savent exactement ce qu'ils sont en mesure de faire et sont aptes à juger de leurs facultés même après 65 ans. Mon expérience avec plusieurs de mes confrères plus âgés confirme sans exception cette affirmation. Cela s'explique aussi par le fait que les médecins se doivent de surveiller eux-mêmes la qualité de leur travail au cours de toute la durée de leur activité professionnelle.

\section{Le flegme est une forme plaisante de la conscience de soi}

(Marie von Ebner-Eschenbach)

$\mathrm{Au}$ contraire des médecins en pratique privée, nos confrères dans les cliniques prennent généralement leur retraite du jour au lendemain. A l'exception de quelques rares professeurs ordinaires à l'université, les médecinschefs doivent arrêter de travailler au plus tard à l'âge de 65 ans révolus, indépendamment du fait qu'ils pourraient encore fournir des prestations de qualité ou souhaiteraient rester à l'œuvre plus tard encore. De qui s'agit-il donc lorsque l'on parle de médecins que l'on «remercie» en raison de leur âge? Ils appartiennent à plusieurs disciplines et ont au moins 30 ans de pratique professionnelle ininterrompue. Même si leur habileté manuelle et leur «efficacité» ont peut-être quelque peu diminué, ils sont encore tout à fait à la hauteur de leur tâche. Ils ne se lèvent probablement plus aussi facilement la nuit et ré- cupèrent moins vite que les autres après une nuit de travail, mais leur grande expérience fait plus que compenser ce léger désavantage. Hormis cette expérience et la somme de connaissances qui l'accompagne, la patience est aussi leur fort. Somme toute, il s'agit d'un potentiel auquel on ne recourt plus et qui de ce fait dépérit. C'est précisément cette somme de connaissances et cette expérience qui seraient pour la formation de la relève d'une énorme importance. Le flegme et le pouvoir d'intuition de ces aînés, dans notre quotidien chahuté, apporteraient un équilibre bienvenu.

\section{Avec l'âge, le discernement augmente et le génie diminue \\ (Emmanuel Kant)}

C'est exactement là l'avantage de la génération plus âgée de médecins expérimentés par rapport à la jeune génération. Si les cliniques engageaient un médecin-chef retraité, à un petit pourcentage d'occupation, pour soutenir la formation postgraduée de la relève, ce serait un excellent investissement. Cela permettrait d'offrir une formation postgraduée mieux structurée à nos jeunes confrères, qui pourraient s'y consacrer de façon plus ciblée et continue. Une solution meilleure encore serait d'offrir des variantes de retraites plus souples qui permettraient à des médecins expérimentés et retraités d'être engagés comme consultants en matière de formation prégraduée et postgraduée, ou dans d'autres domaines cliniques. Il va de soi que l'on pourrait également faire appel à des médecins en pratique privée.

Nous ne souhaitons absolument pas séparer la formation postgraduée du quotidien clinique. Il demeure important que les médecins cadres continuent d'enseigner au jour le jour. Mais le recours à des «maîtres» spécialement choisis rendrait possible une meilleure structure et un approfondissement de la formation postgraduée en vue d'assurer à nos jeunes collègues une continuité de haut niveau. Ceci grâce aux confrères plus âgés et à leur grande expérience professionnelle et humaine!

Dr Brigitte Muff, membre du Comité central de la FMH 